\title{
Diophantine approximation and the solubility of the Schrödinger equation
}

\author{
Simon Kristensen ${ }^{1}$ \\ Department of Mathematics \\ University of York \\ Heslington \\ York, YO10 5DD \\ United Kingdom \\ Tel.: (+44) [0]1904 433072 \\ Fax.: (+44) [0]1904 433071
}

\begin{abstract}
We characterise the set of periods for which number theoretical obstructions prevent us from finding periodic solutions of the Schrödinger equation on a two dimensional torus as well as the asymptotic occurrence of possible resonances.
\end{abstract}

Key words: The Schrödinger equation, Diophantine approximation, Hausdorff dimension

PACS: 03.65.-w, 03.65.Db

2000 MSC: 81Q05, 11K60

\section{Introduction}

Number theoretical considerations arise naturally in the theory of partial differential equations in connection with the notorious problem of small denominators. In this paper, we examine such a problem occurring in the Schrödinger equation for a particle moving on a two-dimensional torus in a potential. We also briefly discuss the corresponding but much more difficult problem associated with the classical wave equation.

Email address: sk17@york . ac . uk (Simon Kristensen).

1 Research funded by EPSRC grant no. GR/N02832/01 
A natural way to study the solubility of partial differential equations on a torus is using Fourier series. Solving these equations for the Fourier coefficients formally yields ratios in which the denominators are on the form

$$
a_{1} x_{1}+\cdots+a_{k} x_{k}+a_{0}
$$

where the $x_{j}$ are dependent on the partial differential equation in question and the $a_{i}$ are drawn from certain subsets of the integers, with each choice of $a_{i}$ 's corresponding to an eigenfrequency. When these denominators become small, the corresponding eigenvalue may become large, depending on the value of the numerator. If too many of the denominators become too small, the Fourier series may fail to converge. Diophantine approximation is a suitable tool for studying the exceptional set in which this happens.

The techniques needed for the resolution of the small denominator problem for the Schrödinger equation on the two-dimensional torus have essentially been obtained in a purely number theoretical setting [1,2,3]. However, those papers made no connection between the number theoretical results and physics. In this paper, the connection between the theory of Diophantine approximation and the Schrödinger equation is made, and the relevant results are adapted to the particular small denominator problem associated with the Schrödinger equation.

\section{Solubility of the Schrödinger equation}

For a single particle on a two dimensional torus with dimensions $\alpha \times \beta$, the Schrödinger equation reads

$$
i \hbar \frac{\partial u(x, y, t)}{\partial t}+\frac{\hbar^{2}}{2 m} \nabla^{2} u(x, y, t)=V(x, y, t) u(x, y, t),
$$

where $V$ denotes a smooth potential on the torus. We assume that the potential is periodic in $t$ with period $\gamma>0$. We are looking for smooth solutions $u$ with the same period in $t$.

We may interpret the above setup as considering a particle moving according to the Schrödinger equation in the compact spacetime given by the three dimensional torus with dimensions $\alpha \times \beta \times \gamma$ and with signature $(+,+,-)$. This spacetime is acausal, as it contains closed timelike curves. Thus, in discussing the solubility of the Schrödinger equation in this setting, we are in fact examining whether or not such a spacetime is consistent with quantum physics and in particular with the Schrödinger equation. In popular terms, we are examining whether or not quantum physics objects to certain types of time machines. 
As we are assuming that both $u$ and $V$ are periodic in all three variables, we may expand them in their Fourier series,

$$
u(x, y, t)=\sum_{a, b, c \in \mathbb{Z}} u_{a, b, c} \exp \left(2 \pi i\left[\frac{a}{\alpha} x+\frac{b}{\beta} y+\frac{c}{\gamma} t\right]\right)
$$

and

$$
V(x, y, t)=\sum_{a, b, c \in \mathbb{Z}} V_{a, b, c} \exp \left(2 \pi i\left[\frac{a}{\alpha} x+\frac{b}{\beta} y+\frac{c}{\gamma} t\right]\right) .
$$

Assume for the moment that we have a solution $u$ to (1) and define the forcing term

$$
f(x, y, t)=u(x, y, t) V(x, y, t)=\sum_{a, b, c \in \mathbb{Z}} f_{a, b, c} \exp \left(2 \pi i\left[\frac{a}{\alpha} x+\frac{b}{\beta} y+\frac{c}{\gamma} t\right]\right)
$$

where

$$
f_{a, b, c}=\sum_{a^{\prime}, b^{\prime}, c^{\prime} \in \mathbb{Z}} u_{a^{\prime}, b^{\prime}, c^{\prime}} V_{a-a^{\prime}, b-b^{\prime}, c-c^{\prime}}
$$

Now, equation (1) becomes

$$
i \hbar \frac{\partial u(x, y, t)}{\partial t}+\frac{\hbar^{2}}{2 m} \nabla^{2} u(x, y, t)=f(x, y, t) .
$$

Consider the corresponding homogeneous equation where $f=0$. In this case, inserting the Fourier series for $u$ in (4) yields the only solution $u=0$.

Integrating on both sides and inserting the Fourier series, we see that $\iiint f d x d y d t=0$ or equivalently $f_{0,0,0}=0$. This follows by the periodicity requirements and the fundamental theorem of calculus. This in turn implies that for the solution $u$, we must have $u_{0,0,0}=0$.

To obtain the required Fourier coefficients for $u$ when $(a, b, c) \neq(0,0,0)$, we insert the Fourier series for $u$ and $f$ in (4) and identify the coefficients to obtain a formula for each of the Fourier coefficients of $u$ in terms of the coefficients for the forcing term $f$,

$$
u_{a, b, c}=-\frac{\gamma}{2 \pi \hbar} \frac{f_{a, b, c}}{\frac{\pi \hbar \gamma}{m \alpha^{2}} a^{2}+\frac{\pi \hbar \gamma}{m \beta^{2}} b^{2}+c} .
$$

We are assuming that a smooth solution does indeed exist, so that (5) makes sense. This gives rise to two sufficient conditions for a solution to (1) to exist. The first condition relates to the numerator in (5). Since both $V$ and $u$ are assumed to be smooth, the Fourier coefficients of the product must decay faster than the reciprocal of any polynomial. This implies that for 
any polynomial $P$,

$$
\left|f_{a, b, c}\right|=\left|\sum_{a^{\prime}, b^{\prime}, c^{\prime} \in \mathbb{Z}} u_{a^{\prime}, b^{\prime}, c^{\prime}} V_{a-a^{\prime}, b-b^{\prime}, c-c^{\prime}}\right|=o\left(P(a, b, c)^{-1}\right) .
$$

where we also require that

$$
f_{0,0,0}=\sum_{a^{\prime}, b^{\prime}, c^{\prime} \in \mathbb{Z}} u_{a^{\prime}, b^{\prime}, c^{\prime}} V_{-a^{\prime},-b^{\prime},-c^{\prime}}=0
$$

Condition ( $\left.\mathrm{C} 1^{\prime}\right)$ is equivalent to the requirement that $\iiint f d x d y d t=0$. These condition relates the wave function to the potential.

The second condition relates to the denominator in (5). The Fourier expansion of $u$ will converge if we have for some $v>0$ and some $K>0$,

$$
\left|\frac{\pi \hbar \gamma}{2 m \alpha^{2}} a^{2}+\frac{\pi \hbar \gamma}{2 m \beta^{2}} b^{2}+c\right|>K \max \left(a^{2}, b^{2}\right)^{-v} \text { for any } a, b, c \in \mathbb{Z}
$$

This condition relates the possible periods in $t$ to the possible dimensions of the tori for which the Schrödinger equation (1) may be solved. If conditions $(\mathrm{C} 1),\left(\mathrm{C}^{\prime}\right)$ and (C2) are satisfied, (2) defines a smooth function on the torus, which satisfies (1). Thus these are indeed sufficient for a solution to exist.

A word about the periodicity requirement on $u$. Inspired by Floquet's Theorem, it is reasonable to conjecture that solutions of the form $e^{\lambda t} u(x, y, t)$ would exist with $u$ periodic. However, on substituting this expression into (1), dividing by $e^{\lambda t}$ and rearranging the terms gives us an equation of the same form as (1) with $V(x, y, t)$ replaced by $V(x, y, t)-i \hbar \lambda$ which $u$ must satisfy in order for the more general function to satisfy the original equation. Thus, the same obstructions exist when we consider solutions of this form. Note also, that for such solutions the topology of the spacetime changes, and we are no longer considering the same physical system. Even so, in this non-compact spacetime, the problem of small denominators persists.

In this paper, we are interested in the small denominator problem associated with the failure of Condition (C2) to hold. This means that in effect, we are studying the partial differential equation given by (4). where $f$ is smooth with $\iiint f=0$. Conversely, all our results apply to equations of that type.

For any $x \in \mathbb{R}$, we let $\|x\|$ denote the distance from $x$ to the nearest integer. 
Let $v>0$. We define the set

$$
\begin{aligned}
\mathcal{E}=\left\{(x, y) \in[0,1]^{2}:\left\|a^{2} x+b^{2} y\right\|\right. & <\max \left(a^{2}, b^{2}\right)^{-v} \\
& \text { for infinitely many } \left.(a, b) \in \mathbb{Z}^{2}\right\} .
\end{aligned}
$$

For any $\left(\frac{\pi \hbar \gamma}{m \alpha^{2}}, \frac{\pi \hbar \gamma}{m \beta^{2}}\right) \in \mathcal{E}$, Condition (C2) fails to hold.

More general forms of the set $\mathcal{E}$ have been studied by Schmidt [3] and Rynne [1]. The latter result was generalised to even more general sets by Dickinson and Rynne [2]. Their results relating to the set $\mathcal{E}$ are summarised in the following theorem.

Theorem 1 (Corollary to [3] and [1]) Let $v>0$. The Lebesgue measure of the set $\mathcal{E}$ is full when $v \leq 1$ and null when $v>1$. When $v>1$, the Hausdorff dimension of $\mathcal{E}$ is $1+2 /(v+1)$.

Furthermore, if we let $\epsilon>0$ and $N(k, v ; x, y)$ denote the number of solutions to

$$
\left\|a^{2} x+b^{2} y\right\|<\max \left(a^{2}, b^{2}\right)^{-v}
$$

with $1 \leq a, b \leq k$, then for almost all $x, y \in \mathbb{R}$,

$$
\begin{aligned}
& N(k, v ; x, y)=\left(\sum_{h=1}^{k} \frac{1}{h^{v}}\right)^{2}+O\left(\sum_{h=1}^{k} \frac{1}{h^{v}}\right)^{1+\epsilon} \\
& = \begin{cases}O(1) & \text { when } v>1 \\
(\log k)^{2}+O(\log k)^{1+\epsilon} & \text { when } v=1 \\
\frac{1}{v+1} k^{2(v+1)}+O\left(\frac{1}{v+1} k^{(v+1)}\right)^{1+\epsilon} & \text { when } v<1 .\end{cases}
\end{aligned}
$$

Note that the final asymptotic formula implies the measure results of the theorem. The theorem characterises the values of $\left(\frac{\pi \hbar \gamma}{m \alpha^{2}}, \frac{\pi \hbar \gamma}{m \beta^{2}}\right)$ for which Condition (C2) fails to hold. Indeed, the set of such points must have measure zero and even Hausdorff dimension 1, as the condition is required to hold for all $v>0$.

In physical terms, The first part of this result states that for most of the spacetimes discussed, the Schrödinger equation may be solved using Fourier series. Furthermore, (7) characterises the number of small denominators occurring for $1 \leq a, b \leq k$. Each such small denominator causes the Fourier coefficient to become large, and thus corresponds to a comparatively large frequency in the spectrum of the wave function. 
Remark 2 A direct but rather lengthy proof of the measure and dimension results of Theorem 1 is possible, using methods from Dodson's paper [4].

A related problem is the problem of characterising the problematic periods in the classical, inhomogeneous wave equation,

$$
\frac{\partial^{2} u(t, x, y)}{\partial t^{2}}-\nabla^{2} u(t, x, y)=f(t, x, y) .
$$

On the one dimensional torus, this was studied by Novák [5] and later in more generality by Fečkan [6] and in even higher generality by Gramchev and Yoshino [7], who obtained necessary and sufficient conditions for solubility of a large class of partial differential equations including the wave equation. However, the problem is unsolved for the two dimensional torus. In this case, the analogue of Condition (C1) disappears, as $u$ does not occur on the right hand side. Condition $\left(\mathrm{C1}^{\prime}\right)$ reduces to the condition that $\iiint f=0$. The Diophantine condition corresponding to Condition (C2) becomes

$$
\left|a^{2} x+b^{2} y-c^{2}\right|>K \max \left(a^{2}, b^{2}\right)^{-v}
$$

for some $v>0$ and some $K>0$ for all $(a, b, c) \in \mathbb{Z}^{3}$ with $(a, b) \neq(0,0)$. From Theorem 1, it follows that the set for which this fails to be the case has measure zero and dimension 1 . However, finding the critical exponent $v$ where the measure of the set associated with the failure of (8) drops from full to null and finding the dimension of this set in the null-region turns out to be very difficult.

It should be noted that since

$$
a^{2} x+b^{2} y-c^{2}=\left(\sqrt{a^{2} x+b^{2} y}-|c|\right)\left(\sqrt{a^{2} x+b^{2} y}+|c|\right),
$$

we may instead of (8) consider the condition

$$
\left|\sqrt{a^{2} x+b^{2} y}-\right| c||>K^{\prime} \max \left(a^{2}, b^{2}\right)^{-v^{\prime}}
$$

for some $v^{\prime}>0$ and some $K^{\prime}>0$ for all $(a, b, c) \in \mathbb{Z}^{3}$ with $(a, b) \neq(0,0)$. This follows as the second term in the product is always positive (in fact it is to the order of $\max (|a|,|b|))$. Unfortunately, analysing the Diophantine condition (9) appears to be no less difficult than the original problem (8).

\section{Acknowledgements}

I thank Todor V. Gramchev for his inspirational comments and insight. I also thank Chris Fewster for pointing out the significance of closed timelike 
curves and Maurice Dodson for his comments on the various drafts of the paper. Finally, I thank the referees for their helpful comments.

\section{References}

[1] B. P. Rynne, Acta Arith., 61(1) (1992) 69.

[2] H. Dickinson and B. P. Rynne, Acta Arith., 93(1) (2000) 21.

[3] W. M. Schmidt, Trans. Amer. Math. Soc., 110 (1964) 493.

[4] M. M. Dodson, Uspekhi Mat. Nauk, 48(5(293)) (1993) 77.

[5] B. Novák, Comment. Math. Univ. Carolinae, 15 (1974) 513.

[6] M. Fečkan, Proc. Amer. Math. Soc., 123(2) (1995) 465.

[7] T. Gramchev and M. Yoshino, Publ. Res. Inst. Math. Sci. 31(3) (1995) 443. 